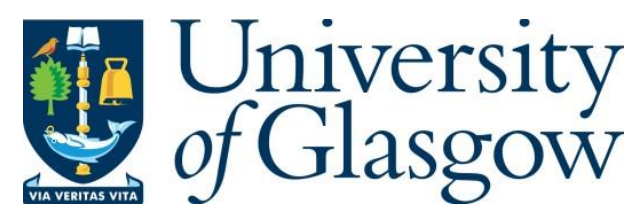

Santos, J.M. et al. (2016) Colloidal Quantum Dot Color Converters for Visible Light

Communications. In: 2016 IEEE Photonics Conference (IPC), Waikoloa, HI, USA, 2-6 Oct 2016, pp. 396-397. ISBN 9781509019069.

There may be differences between this version and the published version. You are advised to consult the publisher's version if you wish to cite from it.

http://eprints.gla.ac.uk/148512/

Deposited on: 20 September 2017

Enlighten - Research publications by members of the University of Glasgow http://eprints.gla.ac.uk 


\title{
Colloidal quantum dot color converters for visible light communications
}

\author{
J. M. Santos, ${ }^{1}$ M. Leitao, ${ }^{1,}{ }^{*}$ C. Foucher,${ }^{1}$ B. Guilhabert, ${ }^{1}$ S. Watson, ${ }^{2}$ A. E. Kelly, ${ }^{2}$ S. Rajbhandari, ${ }^{3,}$ H. Chun, ${ }^{3}$ \\ H. Haas, ${ }^{4}$ G. Faulkner, ${ }^{3}$ D. C. O'Brien, ${ }^{3}$ N. Laurand ${ }^{1, *}$ and M. D. Dawson ${ }^{1}$ \\ ${ }^{1}$ Institute of Photonics, Department of Physics, SUPA, University of Strathclyde, Glasgow \\ ${ }^{2}$ School of Engineering, University of Glasgow, Glasgow, $U K$ \\ ${ }^{3}$ Department of Engineering Science, University of Oxford, Oxford, UK \\ ${ }^{4} \mathrm{Li}$-Fi R\&D Centre, Institute for Digital Communications, University of Edinburgh, Edinburgh, UK
}

\begin{abstract}
The properties and performance of CdSSe/ZnS colloidal quantum dot composite materials for use as InGaN LED color converters in visible light communications applications is reported. $500 \mathrm{Mb} / \mathrm{s}$ optical wireless transmission is demonstrated.

\section{INTRODUCTION}

The electronic and optical properties of colloidal quantum dots (CQDs) make them appealing for color conversion of InGaNbased LEDs [1]. In particular, CQDs possess key advantages over phosphors, typically used in the down-conversion of blue-emitting InGaN LEDs, for applications in visible light communications (VLC), where LEDs are used to transmit data. The shorter excited state lifetimes of CQDs mean they should perform better at higher modulation speeds [2,3] while their wavelength versatility and narrow emission spectrum pave the way for color multiplexing as a way to further increase data rates.
\end{abstract}

Recently, a single blue-emitting microsize LED (microLED) was used to demonstrate VLC at several Gb/s [4]. However, high-speed VLC $(>100 \mathrm{Mb} / \mathrm{s})$ at longer wavelengths using CQDs has not yet been achieved [2]. In this context, we study a CQD/Polymethyl methacrylate (PMMA) composite material for color converting a blue microLED. Color-conversion at different wavelengths is demonstrated and the effect of the CQD concentration on the converted light properties is discussed. It is shown that VLC at several hundreds $\mathrm{Mb} / \mathrm{s}$ data rates is achievable with CQDs.

\section{MATERIALS AND METHODOLOGY}

The CQDs used in this work are green emitting (intrinsic emission at $540 \mathrm{~nm})$ and red emitting $(630 \mathrm{~nm})$ alloyedcore/shell CdSSe/ZnS nanocrystals with a $6 \mathrm{~nm}$ mean diameter. Engineering of the energy levels in such CQDs is obtained by changing the alloy composition of the core. To form the composite, the CQDs are incorporated into a PMMA matrix at different CQD-to-PMMA weight ratio $(1 \%$ and

\footnotetext{
${ }^{\S} S . R$. is now at the University of Coventry

*Corresponding authors: miguel.leitao@strath.ac.uk and nicolas.laurand@strath.ac.uk
}

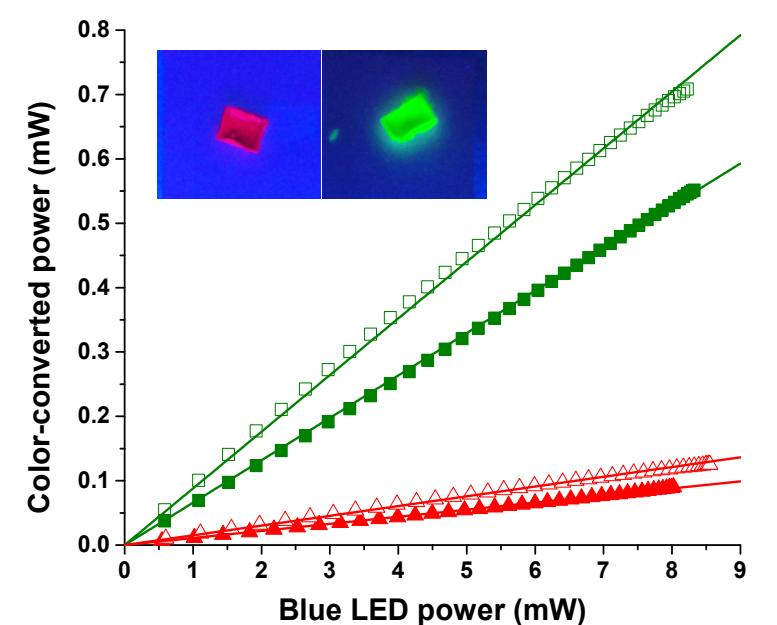

Fig. 1: Color-converted power vs. LED power for $1 \%$ (filled squared) and $10 \%$ (open square) green CQDs and for $1 \%$ (filled triangles) and $10 \%$ (open triangles) red CQDs. Inset: Red and green $C Q D$ composite samples under UV illumination.

$10 \%$ ). The nominal thickness of the CQD/PMMA converters under study is $1 \mathrm{~mm}$. Two such converters can be seen in the inset of Fig. 1.

The color converters are remotely excited with a square $100 \times 100 \mu \mathrm{m}^{2}, \quad 450 \mathrm{~nm}$-emitting microLED [5] for the characterization of the forward conversion spectrum, power efficiency and optical modulation bandwidth. Forward converted emission includes the losses of the collecting optics and is detected under continuous-wave microLED excitation with a power meter or a spectrometer. For the modulation bandwidth measurements, the microLED is dc-biased and modulated with a $0.25 \mathrm{Vpp}$ frequency-swept signal while the detection is done with a high-speed photodiode $(1.4 \mathrm{GHz}$ bandwidth). Photoluminescence (PL) lifetimes are also measured using time-correlated single photon counting (Edinburgh Instruments).

VLC in free space over a meter is demonstrated using twolevel pulse amplitude modulation (PAM). In this case a pulse function arbitrary generator provides the data stream to the microLED and the detection of the color-converted light is done with an avalanche photodiode. The received signal is post-processed using a Matlab script. More details about the VLC experiment can be found in [6]. 


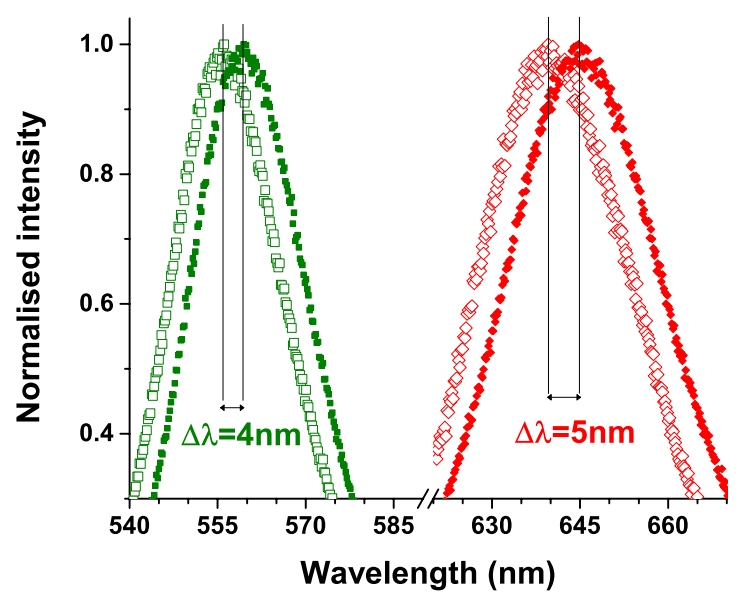

Fig. 2: (Left) Color-converted spectra for the 1\% (open square) and 10\% (filled squares) green CQD composite. (Right) 1\% (open diamonds) and $10 \%$ (filled diamonds) red CQD composite. Note the break in the $x$ axis.

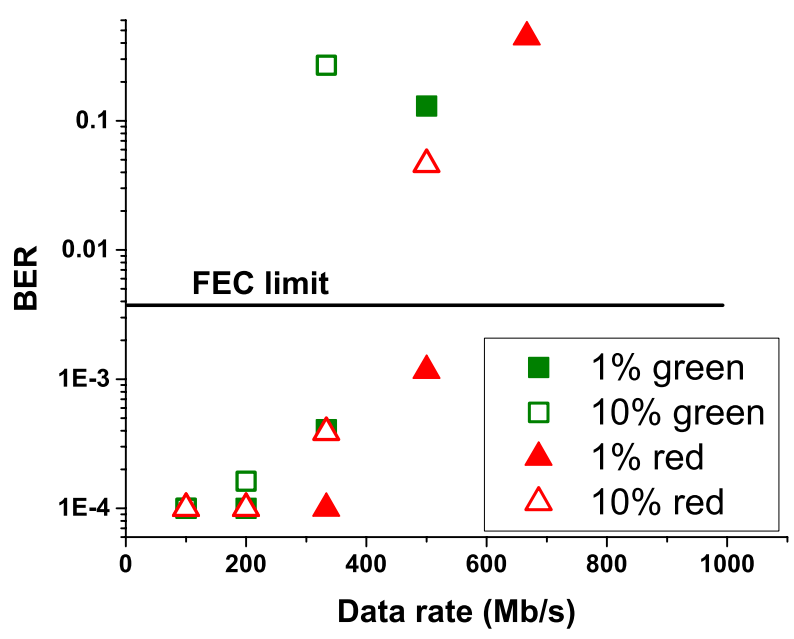

Fig. 3: BER vs. data rates for VLC experiments using 2-PAM.

\section{RESULTS AND DISCUSSIONS}

The plots of Fig. 1 represent the forward emitted light converted by the CQD samples vs. the incident microLED power. In terms of raw power conversion, the samples with the highest CQD concentration (10\%) perform better, simply because they absorb more of the incident LED light. The efficiency of the samples, defined as the ratio of the forward color converted light (collected by the system) by the absorbed microLED power, is $8+/-2 \%$ for the green CQDs and $1.5+/ 0.5 \%$ for the red CQDs. The higher efficiency of the green CQDs is attributed to the different alloy composition of the core that leads to a lower lattice mismatch with the $\mathrm{ZnS}$ shell than in the case of the red CQDs.

The spectra of the converted light are shown in Fig. 2. For both green and red CQDs, the spectrum shifts to longer wavelengths at higher CQD concentration because of self- absorption. The shift between the sample with $1 \%$ and $10 \%$ CQD concentration is around $5 \mathrm{~nm}$. The $1 \%$ samples are already red shifted significantly when compared with more diluted samples (data not shown) with the intrinsic peak emission of the green and red CQDs at, respectively, $540 \mathrm{~nm}$ and $630 \mathrm{~nm}$. Interestingly, the optical modulation bandwidths of the CQD samples are lower at higher CQD concentration: $15 \mathrm{MHz}$ and $12 \mathrm{MHz}$ for, respectively, the $1 \%$ and $10 \%$ green samples, and $27 \mathrm{MHz}$ vs $17 \mathrm{MHz}$, respectively, for the $1 \%$ and $10 \%$ red samples. Measured PL lifetimes are consistent with these bandwidth values: respectively $16 \mathrm{~ns}$ vs. $22 \mathrm{~ns}$ for the green CQDs and $10 \mathrm{~ns}$ vs. $15 \mathrm{~ns}$ for the red CQD samples. This 'slowing down' of the color-conversion dynamics is attributed to the self-absorption effect, which is also at the origin of the spectral shift and is significant at higher CQD concentration.

The bit-error rate (BER) as a function of the data rate for VLC demonstrations using 2-PAM modulation is plotted in Fig. 3. The BER limit is $3.8 \times 10^{-3}$, considering forward error correction (FEC) could be applied. It is seen that the sample that performs the best under these conditions is the $1 \%$ red CQD samples, i.e. the color converter that has the highest modulation bandwidth, with a data rate above $500 \mathrm{Mb} / \mathrm{s}$. The green CQD samples are more efficient but their slower modulation bandwidths means that under the same conditions the achievable data rate is a bit lower, close to $400 \mathrm{Mb} / \mathrm{s}$. These results are consistent with both bandwidth and PL lifetime measurements. While the PL lifetimes of CQDs is their limiting factor for such application, their efficiency and narrow emission linewidths means that $>1 \mathrm{~Gb} / \mathrm{s}$ transmission should be possible given these results.

\section{CONCLUSION}

In summary, we have demonstrated that CQDs could be utilized for color-conversion of InGaN microLEDs in VLC applications. We have reported on the properties of a format of CQD composite, operation at two different wavelengths, and discussed the effect of CQD concentration on the spectral and dynamics properties of the color-converters. $500 \mathrm{Mb} / \mathrm{s} \mathrm{VLC}$ at a single wavelength was reported.

\section{ACKNOWLEDGMENT}

This work was supported by the EPSRC programme Grant 'Ultra-parallel visible light communications', EP/K00042X/1. The authors acknowledge Dr. E. Xie for the microLED fabrication. Supporting data is available:

DOI: $10.15129 / 6440 \mathrm{a} 143-7255-40 \mathrm{cf}-\mathrm{a} 214-44 \mathrm{a} 79 \mathrm{fcf} 11 \mathrm{c} 6$

\section{REFERENCES}

[1] T. Erdem and H. V. Demir, Nanophotonics 2, 1, pp. 57-81 (2013).

[2] N. Laurand et al., Optical Material Express 2, 3, pp. 250-260 (2012).

[3] J. Grubor et al., J. Lightwave Technol. 26, 24, pp. 3883-3892 (2008).

[4] D. Tsonev et al., IEEE Photonics Technol. Lett. 26, 7, pp. 637640 (2014).

[5] Z. Gong et al., J. Appl. Phys. 107, 013103 (2010).

[6] D. C. O'Brien et al., J. Light. Technol. 30, pp. 2181-2187 (2012). 